\title{
Variations in the Impact Spatter Patterns due to Blunt Weapons Inflicted by Different Gender and Age Groups
}

\author{
Wafa $\mathrm{K}^{1 *}$, Feras $\mathrm{KS}^{3}$, Ashiq $\mathrm{MI}^{3}$, Butt $\mathrm{M}^{1}$, Khan \\ $\mathbf{A A}^{1}$, Aslam MJ ${ }^{1}$, Nadir $\mathbf{H}^{1}$ and Tahir $\mathbf{M A}^{2}$ \\ ${ }^{1}$ Department of Institute of Molecular Biology and \\ Biotechnology, The University of Lahore, Pakistan \\ ${ }^{2}$ Director General of Punjab Forensic Science Agency \\ Lahore, Pakistan \\ ${ }^{3}$ Department of Trace Chemistry, Punjab Forensic Science \\ Agency Lahore, Pakistan \\ *Corresponding author: Komal Wafa, Department \\ of Institute of Molecular Biology and Biotechnology, The \\ University of Lahore, 1-Km Raiwind Rd, Lahore, Pakistan
}

Received: April 30, 2021; Accepted: June 01, 2021; Published: June 08, 2021

\begin{abstract}
Bloodstain pattern analysis makes use of the mechanics of bloodstains to estimate the time, nature and the sequence of events at the crime scene. In this study, a quantitative methodology was developed which includes manually calculating the average size of the bloodstains and the synthetic bloodstains to distinguish the impact stain patterns created by different gender and age groups using the two blunt weapons. Impact stains were created by hitting sponges soaked in blood and synthetic blood using a hammer and a cricket bat. A smaller bloodstain size was observed of the group of males aged between 18 to 30 years. Greater amount of spatter and the distribution of the blood spatter were observed with the bat as compared to hammer due to larger surface area of bat. Cast off patterns were studied and were observed to be produced only by the bat which can be attributed to its higher surface area. Results showed difference in the bloodstain diameter produced from human blood and synthetic blood evaluating if the synthetic blood is suitable for use, as well as variation in the average stain size between produced by the four groups using both the fluids.
\end{abstract}

Keywords: Impact; Bloodstain; Blunt; Crime; Cast-off patterns; Spatter

\section{Abbreviations}

ABFO: American Board of Forensic Odontology; H.B: Human Blood; S.B: Synthetic blood; WOP: Weapon of Offense; LW1: Left Wall 1; LW2: Left Wall 2; RW1: Right Wall 1; RW2: Right Wall 2; FWL: Front Wall Left; FWR: Front Wall Right; FF: Floor Front; FR: Floor Rear; C1: Ceiling 1; C2: Ceiling 2; C3: Ceiling 3; PPE: Personal Protective Equipment; EDTA: Ethylenediaminetetraacetic Acid

\section{Introduction}

Blood is a type of physical evidence and is considered among one of the primary evidence in violent crime scenes. The analysis of blood and the stains is an important aspect in forensic investigations of the crime scenes in which they are present [1]. Bloodstain pattern analysis is the observation of shapes and the classification and distribution of patterns of bloodstain in order to provide an understanding of the physical events of an offence that gave rise to the creation of those stains [2].

A correct interpretation of bloodstains at a scene of a crime is of great importance in the reconstruction of that crime scene and to estimate and establish a sequence of events including area of crime scene, acts of victims and suspects, their role in crime scene and actions after committing crime [3]. There is a vital need to interpret bloodstain patterns in forensic science based on observed, objective and reproducible properties [4].

Bloodstains found on a crime scene, can help us in determining who was involved in an offense by using various biological techniques, the bloodstain pattern analysis on the other hand, can help to identify where and how the particular occurred [5]. Bloodstain patterns are also considered reconstructive evidence of great significance in homicide cases [6]. These patterns are categorized into three categories which include passive stains, transfer stains and projected stains. The projected stains are also referred to as impact stains. They are further divided into three groups based on the amount of velocity forcing that bloodstain which include low velocity, medium velocity and high velocity impact spatters [7].

Studies have been performed in this domain of forensic science, Bandyopadhyay and Basu studied transfer bloodstain patterns of blunt weapon hammer by conducting experiment on hollow coconut with hair wigs to simulate the head hit [8].

A pattern of bloodstain resulting from blood drops generated from an object because of its motion is called cast off pattern [9]. Analysis of cast-off pattern plays an important role in forensic criminal investigations because they suggest a minimum number of blows during murder as a result of the beating incident hence they cannot indicate the total number of blows delivered during an incident [10].

James Alan Fox and Emma E. Fridel analyzed homicide cases from 1976-2015 in which they examined gender and age differences in offenders and victims. They found that in most of the cases, people of age 18-24 and above 25 years were involved [11].

Impact patterns are significant in the reconstruction of the crime scene, and it is important to understand the mechanics involved in their creation [12]. The current study was designed to analyze the patterns of bloodstains produced and how the information could be extracted out of those patterns which can aid in narrowing down the investigation and search in crime scenes similar to that which is reconstructed in the study, with regard to the gender and age group of the people, also the nature and surface area of the weapon used to produce the blood spatter. Synthetic blood was also made and used to generate the impact spatter in this study for comparison with human 
bloodstains. The viability of the use of synthetic blood for training and demonstrative purposes was also calculated by comparative analysis with the human bloodstains.

\section{Materials and Methods}

A total of twelve, six males and six females of ages 18-45 years participated in this experiment. Two blunt weapons i.e. a hammer and a cricket bat (both having different surface areas) were used to produce both the blood and synthetic bloodstain patterns by striking fluid-soaked sponges. An overview of the methodology used to generate impact spatter is shown in (Figure 1).

The spatter patterns examined in this study were produced in a controlled environment in the Punjab Forensic Science Agency (PFSA), Lahore-Pakistan. The area dedicated for the crime scene reconstruction was used to perform this experiment (Figure 2).

White paper sheets were used as the target surface over which the blood patterns and the synthetic bloodstains patterns were collected, recorded and analyzed. The walls and the ceiling of the room were covered with paper as well to record the patterns that were created on the ceiling. A total 48 sponges were used in the experiment. Three pints of blood were collected from Mayo Hospital, Lahore. Ethylenediaminetetraacetic Acid (EDTA) of Fischer scientific brand was added to the blood and it was refrigerated at $4^{\circ} \mathrm{C}$. The sponges were soaked with two different types of fluids (human and synthetic blood). At once instance one sponge was soaked in the blood to perform the experiment. When the experiment was performed with the blood-soaked sponge and the stains dried, it was replaced by the sponge which was soaked in the synthetic blood. Blue and

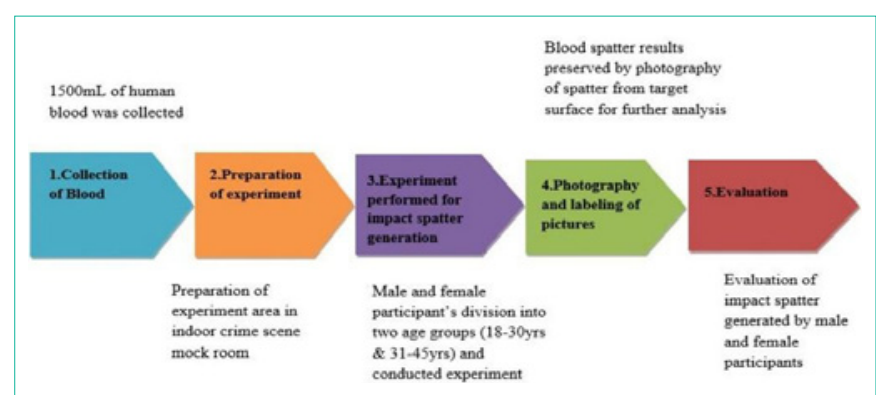

Figure 1: An overview of quantitative methodology for generating impact bloodstain patterns.

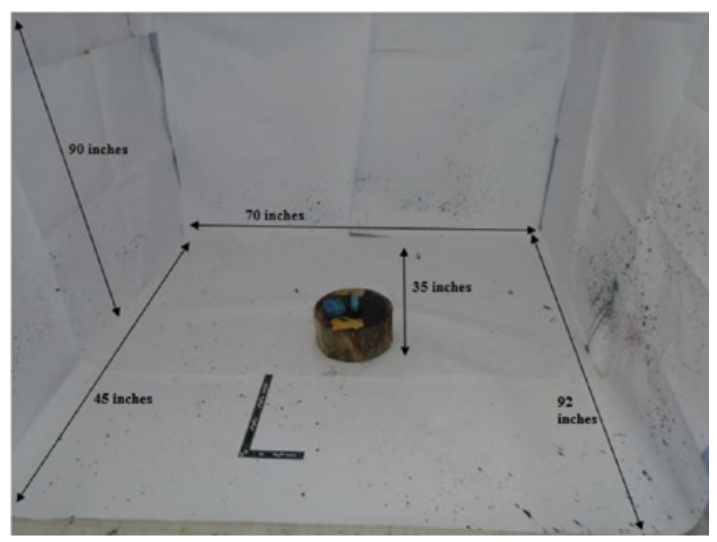

Figure 2: Experimental area covered with white paper sheets.

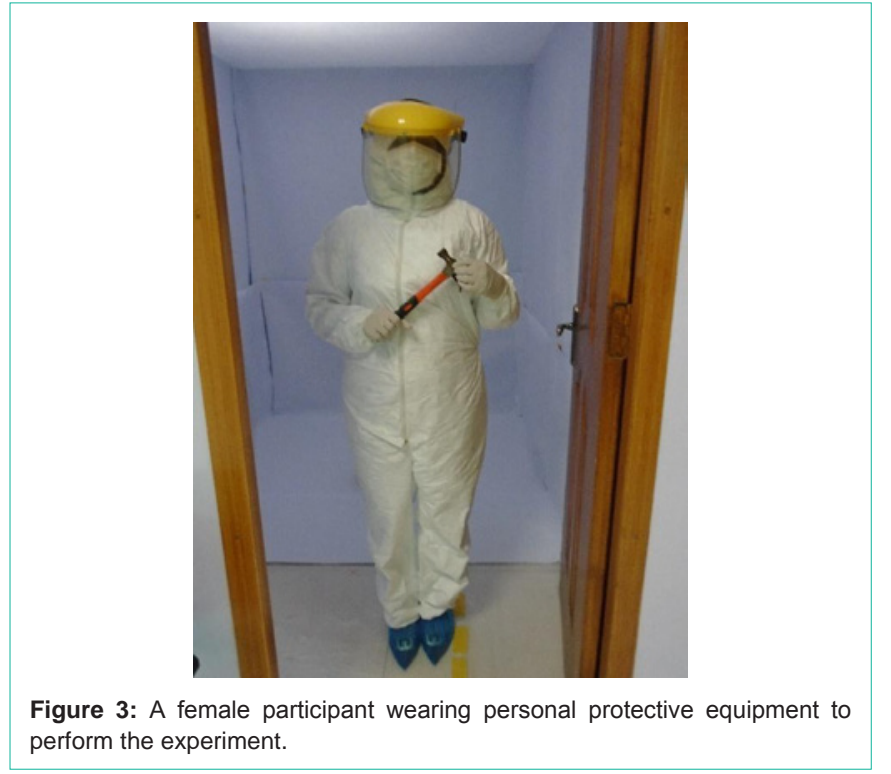

green liquid food colors were used as one of the ingredients to make synthetic blood. These two colors were used in order to differentiate the synthetic blood from the blood. Synthetic blood of blue color was used for the experiment performed with bat while the synthetic blood of green color was used when performing the experiment with hammer.

Synthetic blood was formed by mixing the chemical reagents amylum (corn starch), a product of Hassani Food Industries, Xtore glucose syrup (corn syrup) obtained from Baking shop, liquid food color (blue and green) obtained from Foster Clarks and water [13]. Density of both fluids was calculated. Synthetic blood showed the density $1.4 \mathrm{~g} / \mathrm{ml}$ while human blood's density was $1.9 \mathrm{~g} / \mathrm{ml}$. The sponge used was measured and was 3.5 inches in length, 1.3 inches in thickness and 2.5 inches in width.

For the experiment, the 12 participants were divided into two age groups (18-30 years and 31-45 years). Detail of the participants is mentioned in (Supplementary Table 1). Each age group of the two genders had three participants. All of the participants performed the experiment separately with both the blunt weapons by hitting the sponges soaked in the two different types of fluids.

For the experiment, each participant wore Personal Protective Equipment (PPE) (Figure 3) and first performed the experiment using hammer by hitting the sponge. For each participant, a sponge soaked in $50 \mathrm{ml}$ of human blood was placed on a wooden pedestal of height of 6.5 inches and a diameter of 10 inches. Each participant hit the sponge designated for them 5 times using the hammer to create impact spatter while keeping the distance of 25 inches from wooden pedestal (Figure 4). The blood spatter produced on the surface was captured using a digital camera; Sony Cyber-shot DSC-W800. After recording the blood spatter, the spatter created using synthetic blood was also generated similarly, for this however, a sponge which was soaked in $50 \mathrm{ml}$ synthetic blood was used. After initial observation of the stains produced by both the fluids, that particular set of sheets were removed and replaced with a new set of sheets for the next participant to perform the same experiment. All the participants 


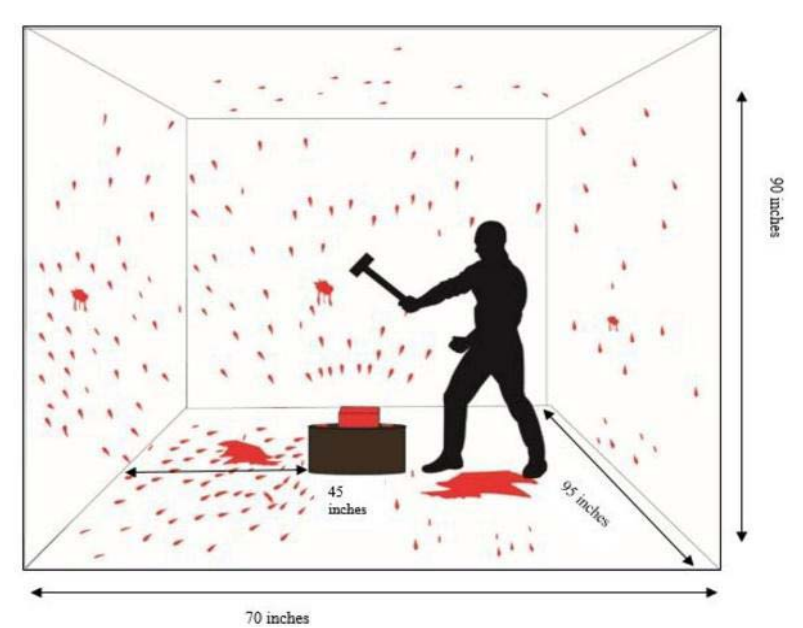

Figure 4: 3 Dimensional representation of blunt weapon hammer infliction to the target sponge.

Table 1: Blunt weapons used in the study along with information about length, weight and surface area.

\begin{tabular}{|c|c|c|c|}
\hline Blunt weapon & Weight(g) & Length(cm) & Surface area \\
\hline Hammer & $453.592 \mathrm{~g}$ & $27.178 \mathrm{~cm}$ & $3.79 \mathrm{~cm}^{2}$ \\
\hline Bat & $793.787 \mathrm{~g}$ & $85.09 \mathrm{~cm}$ & $77.5 \mathrm{~cm}^{2}$ \\
\hline
\end{tabular}

performed the experiment using the hammer similarly.

Similarly, when the experimentation using hammer was complete, the participants performed the same experiment using bat as the weapon to generate the spatter instead of hammer. After the experiment with bat, the white sheets with impact spatter patterns were observed and recorded just as it was done earlier when the experiment was performed using hammer. The surface area of the two weapons, the length and the weight was also calculated as well of the two weapons which were used in this study (Table 1).

\section{Results}

After the experiment, the resultant impact spatter generated was recorded on the white chart paper that covered the walls, ceiling and the floor.

\section{Measurement of bloodstain and synthetic bloodstain size}

Bloodstain size produced by each participant was manually measured with calibrated ABFO scale. In this study, diameter of approximately 15,360 bloodstains was calculated. Females aged between 18-30 years produced the largest bloodstain size with the average of 5.59 and $3.5 \mathrm{~mm}$ using hammer and bat respectively, in comparison, the male participants of the same age group generated the smallest bloodstain size of 1.77 and $1.47 \mathrm{~mm}$ using hammer and bat respectively. The average bloodstain size produced by males of the age group 31 to 45 was greater than females of the same age group (Table 2).

In case of synthetic blood used, females showed greater average bloodstain size as compared to males regardless of the age group and the weapon (Table 3). Chi- Square Test was applied on bloodstain values to evaluate them statistically (Table 4).

\section{Evaluation of distribution of bloodstains and synthetic bloodstains with respect to the area}

Distribution of bloodstains into the whole area of experiment is mentioned in (Supplementary Table 2). Supplementary Table 2 shows impact blood spatter patterns formed with human and synthetic blood on eight sheets Left Wall 1 (LW1), Left Wall 2 (LW2), Right Wall 1 (RW1), Right Wall 2 (RW2), Front Wall Left (FWL), Front Wall Right (FWR), Floor Front (FF) and Floor Rear (FR), using hammer and bat by both the genders.

Table 5 shows bloodstain size of cast-off patterns formed on three ceiling sheets (C1, C2 and C3). A total of three cast-off linear patterns were observed on ceiling sheets that were produced by male participants' strike with bat. Therefore, minimum number of blows can be calculated by putting value in number of blows equation:

Minimum Number of Blows $=n+1=3+1=4$.

Above equation is the review of $n+1$ formula for calculation of cast-off pattern using minimum number of blows and different weapon of offense. In the experiment, the total number of blows was 05 and cast-off patterns, when produced were seen to be produced after third strike. Cast-off patterns were observed in the case of two male participants from 18-30 years' age group and one male participant from 31-45 year age group.

Using synthetic blood, male participants from age group 18-30 years' produced cast-off stains on sheets $\mathrm{C} 1$ while with human blood cast-off stains were observed on sheet C2. No cast-off pattern was observed on sheet C3. Using human blood, male participants of age group 31-45 years' produced cast-off stains on sheets C1 and C2 while on sheet C3 cast-off stain was only produced using synthetic blood.

Participants from both age groups showed different patterns due to difference in the angle of swing, difference in the height, the angle

Table 2: Bloodstain size in gender \& age groups produced with human blood by using blunt weapons.

\begin{tabular}{|c|c|c|c|c|}
\hline \multicolumn{4}{|c|}{ Average bloodstain size with human blood } \\
\hline Weapon of offense & $\mathbf{1 8 - 3 0}$ yrs (female) & $\mathbf{3 1 - 4 5}$ yrs (female) & $\mathbf{1 8 - 3 0}$ yrs (male) & $\mathbf{3 1 - 4 5}$ yrs (male) \\
\hline Hammer & $5.59 \mathrm{~mm}$ & $1.8 \mathrm{~mm}$ & $2.71 \mathrm{~mm}$ \\
\hline Bat & $3.5 \mathrm{~mm}$ & $1.88 \mathrm{~mm}$ & $1.47 \mathrm{~mm}$ \\
\hline
\end{tabular}

Table 3: Bloodstain size in gender \& age groups produced with synthetic blood by using blunt weapons.

Table 3: Bloodstain size in gender \& age groups produced with synthetic blood by using blunt weapons.
\begin{tabular}{|c|c|c|c|c|}
\hline \multicolumn{3}{|c|}{ Average bloodstain size with synthetic blood } \\
\hline Weapon of offense & $\mathbf{1 8 - 3 0}$ yrs (female) & $\mathbf{3 1 - 4 5}$ yrs (female) & $\mathbf{1 8 - 3 0}$ yrs (male) & $\mathbf{3 1 - 4 5}$ yrs (male) \\
\hline Hammer & $2.95 \mathrm{~mm}$ & $2.6 \mathrm{~mm}$ & $1.26 \mathrm{~mm}$ & $1.12 \mathrm{~mm}$ \\
\hline Bat & $2.0 \mathrm{~mm}$ & $1.3 \mathrm{~mm}$ & $1.4 \mathrm{~mm}$ \\
\hline
\end{tabular}


Table 4: Chi-squares values of bloodstains.

\begin{tabular}{|c|c|}
\hline Chi Square Observations & \\
\hline \multicolumn{2}{|c|}{ For human bloodstains } \\
\hline \multicolumn{2}{|c|}{$\mathrm{H}_{\mathrm{o}}=$ The two weapons used by different gender/age groups in this experiment will produce bloodstains of different size using human blood. } \\
\hline Total number of observations (n) & 2 \\
\hline Degree of freedom (n-1) & $2-1=1$ \\
\hline $\begin{array}{l}\text { Critical value of Chi- square distribution for } 1 \text { degree of freedom at } 0.05 \\
\text { significance level }\end{array}$ & $X^{2}=3.841$ \\
\hline Calculated value of Chi- square & 0.973 \\
\hline Conclusion & $\begin{array}{l}\text { Calculated value of Chi- square }<\mathrm{X}^{2} \text { Critical value, hence null hypothesis }\left(\mathrm{H}_{\mathrm{o}}\right) \\
\text { can't be rejected }\end{array}$ \\
\hline \multicolumn{2}{|c|}{ For synthetic bloodstains } \\
\hline \multicolumn{2}{|c|}{$\mathrm{H}_{\mathrm{o}}=$ The two weapons used by different gender/age groups in this experiment will produce bloodstains of different size using synthetic blood. } \\
\hline Total number of observations (n) & 2 \\
\hline Degree of freedom $(n-1)$ & $2-1=1$ \\
\hline $\begin{array}{l}\text { Critical value of Chi- square distribution for } 1 \text { degree of freedom at } 0.05 \\
\text { significance level }\end{array}$ & $X^{2}=3.841$ \\
\hline Calculated value of Chi- square & 0.905 \\
\hline Conclusion & $\begin{array}{l}\text { Calculated value of Chi- square }<\mathrm{X}^{2} \text { Critical value, hence null hypothesis }\left(\mathrm{H}_{\mathrm{o}}\right) \\
\text { can't be rejected }\end{array}$ \\
\hline
\end{tabular}

Table 5: Calculated Cast-off Stain Size at the Ceiling Sheets (C1, C2 \& C3) in Experimental Area.

\begin{tabular}{|c|c|c|c|}
\hline Sheets & C1 & C2 & C3 \\
\hline Area of sheets $\left(\mathrm{ft}^{2}\right)$ & 15.5 & 15.5 & 15.5 \\
\hline Average stain size with respect to participants \\
\begin{tabular}{|c|c|c|c|}
\hline With Bat as Blunt Weapon \\
\hline $\mathbf{1 8 - 3 0}$ male & S.B $=2.5 \mathrm{~mm}$ & H.B $=9.2 \mathrm{~mm}$ & \\
\hline $\mathbf{3 1 - 4 5}$ male & H.B $=1 \mathrm{~mm}$ & H.B $=2.7 \mathrm{~mm}$ & S.B $=19 \mathrm{~mm}$ \\
\hline
\end{tabular}
\end{tabular}

${ }^{*}$ H.B.: Human Blood; 'S.B.: Synthetic Blood.

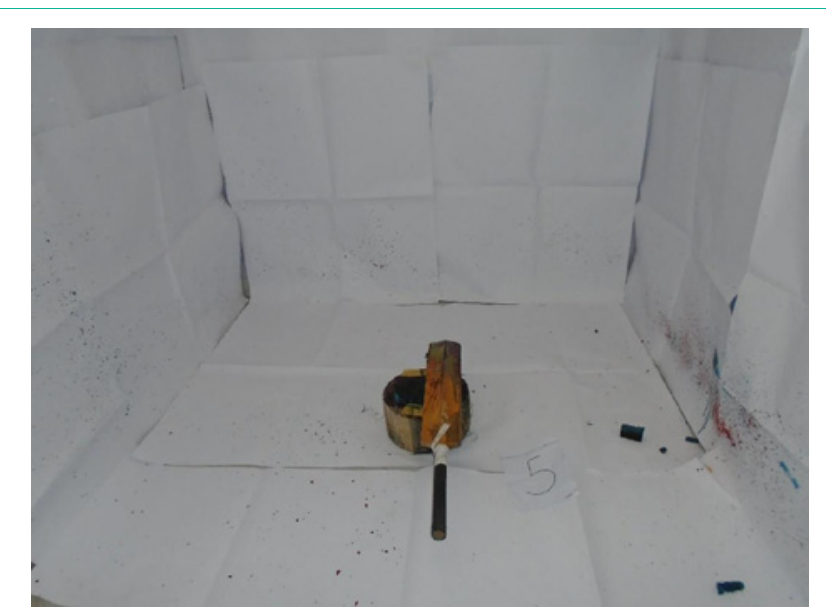

Figure 5: Impact spatter produced by a male participant aged between 31-45 years using bat.

of strike and the force, can be considered as one of the factors as well.

No cast-off pattern was observed in case of female participants using any of the two fluids and the weapons which were used in this experiment. No cast-off patterns were produced when the experiment was performed by males using the hammer.

Amount of spatter and bloodstain size and synthetic blood stain

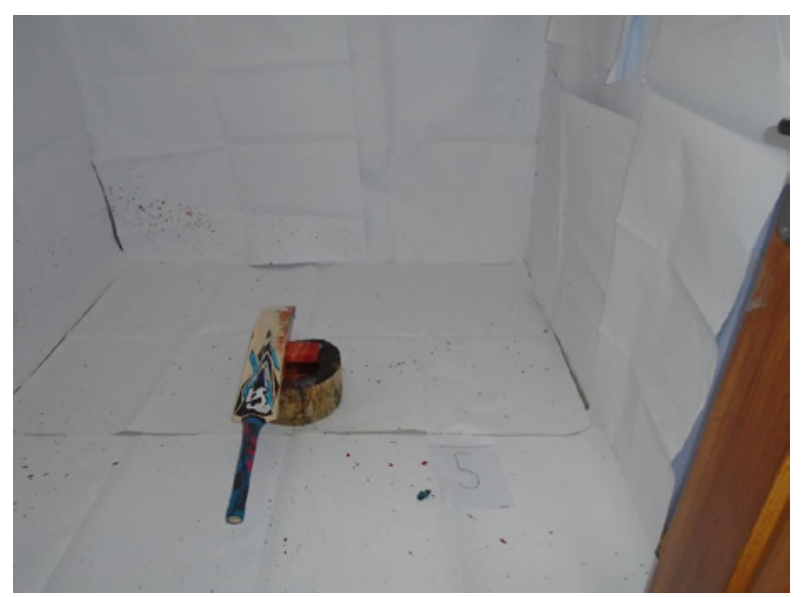

Figure 6: Impact spatter produced by female participant aged between 31-45 years using bat.

size produced by different gender and age groups. Six females and six males of the mentioned age groups hit the blood-soaked sponge using blunt weapons from standing position. The resulting impact blood spatter patterns were studied and it was determined that male participants produced more blood spatter than females using bat after administrating 5 hits to the target as shown in (Figure 5 and Figure 6). It was also observed that the male participants of age group 1830 produced more spatter with small stain size (Figure 7 and Figure 8). Cast-off patterns were only observed on the sheets of three male participants when using bat as a blunt weapon for striking the sponge.

The value of mean for human bloodstains and synthetic bloodstains is shown in (Figure 9). Comparatively, the female participants showed greater stain size using hammer in contrast to stain size generated with bat. From the collected data it can be deduced that males can have a higher impact force thus producing smaller stain size. 


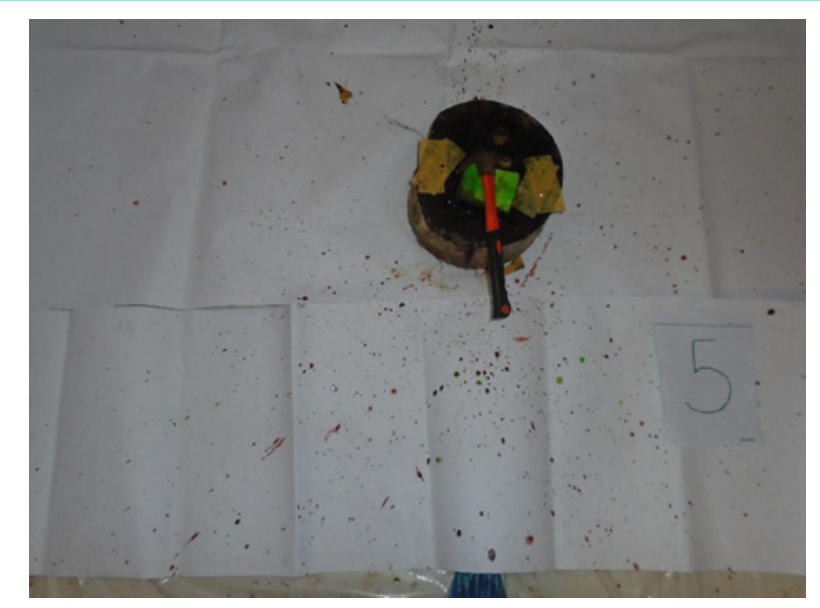

Figure 7: Impact spatter produced by a male participant aged between 18-30 years using hammer.

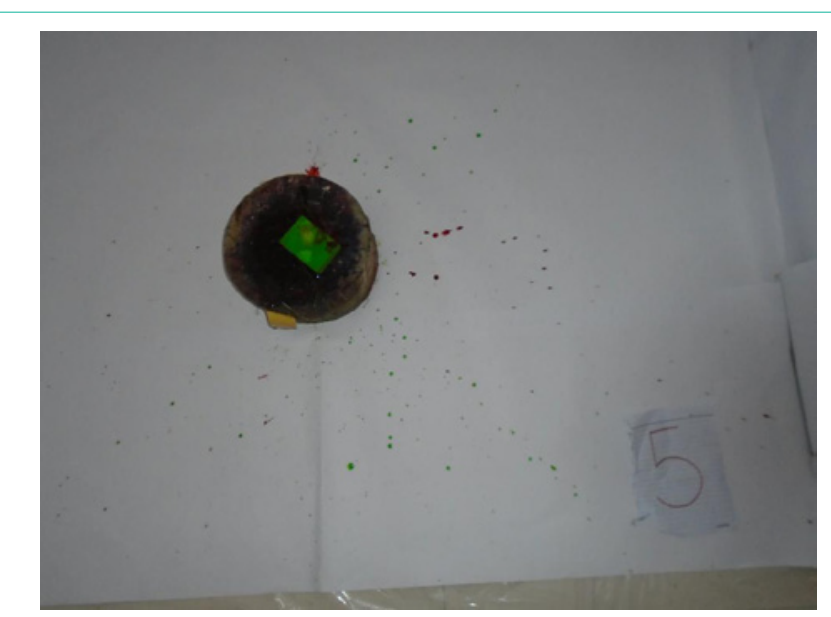

Figure 8: Impact spatter produced by a female participant aged between 1830 years using hammer.

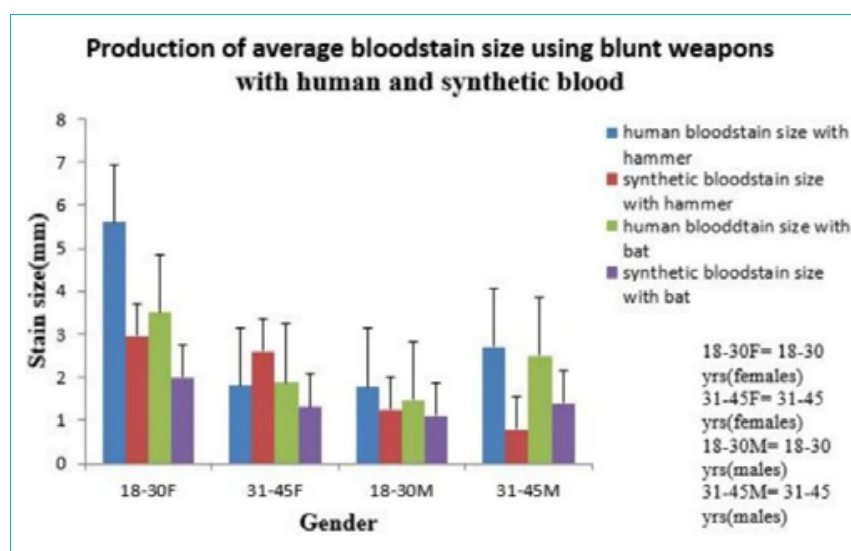

Figure 9: Stain size and gender group relationship with blunt weapons using human blood and synthetic blood.

\section{Effect of fluid type on the shape of spatter}

It was seen that the human blood stains displayed more spikes and spines while stains generated using synthetic blood relatively smoother (Figure 10a and 10b). After drying, the patterns of stains
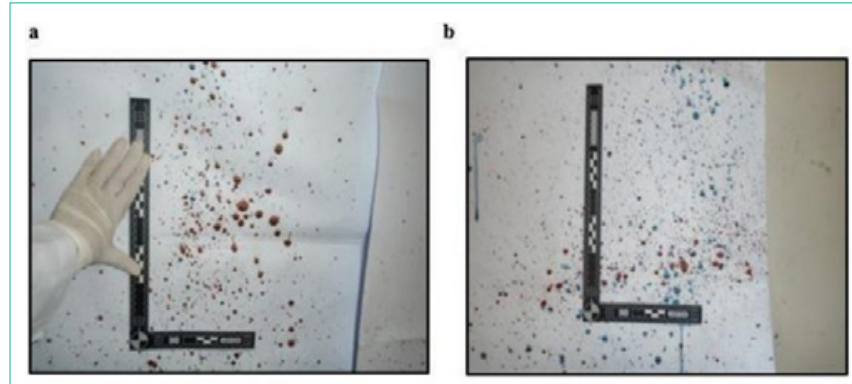

c

d
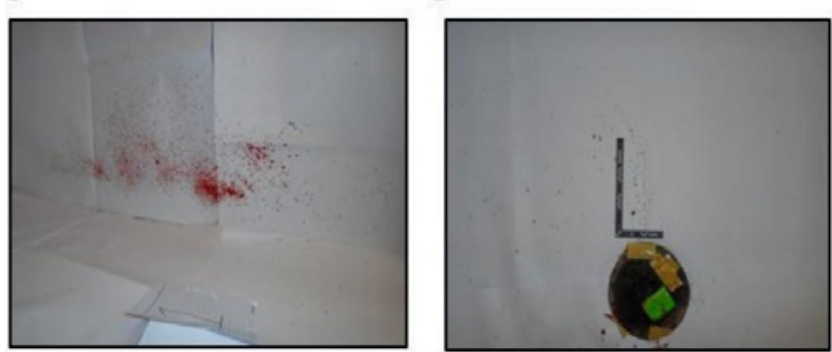

Figure 10: Impact spatter produced with blunt weapons using human blood and synthetic blood. (a) Impact spatter produced with bat using human blood and synthetic blood. (b) Tacky appearance of blue synthetic blood spatter produced with bat. (c) Mist like impact spatter produced with bat. (d) Large circular bloodstains produced with the impact of hammer on the sponge.

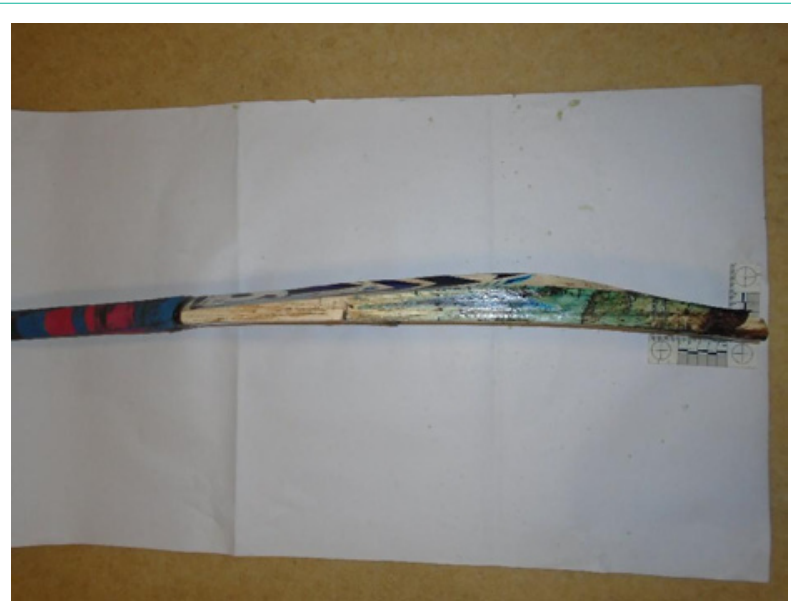

Figure 11: Measurement of surface area of bat with ABFO scale.

generated with synthetic blood appeared irregular as compared to human blood that remained smooth. The stains produced using the hammer were circular and larger than the stains produced using the bat (Figure 10c and 10d).

\section{The relationship among surface area of blunt weapon and amount of spatter}

The relationship between the surface area of blunt weapons (Figure 11 and 12) and the amount of blood spatter was analyzed. The surface area of bat was greater than surface area of hammer and total amount of blood spatter produced by bat was higher in quantity than that produced using hammer, which is an indicative of that there is generally a linear relation between the surface area of weapon and the amount of spatter produced. 


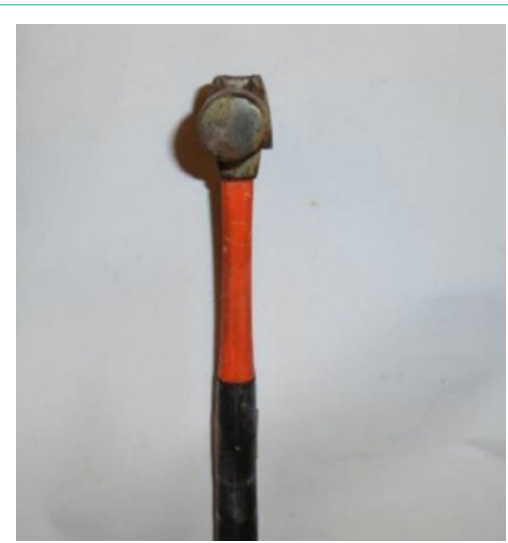

Figure 12: Round surface area of hammer used in the experiment.

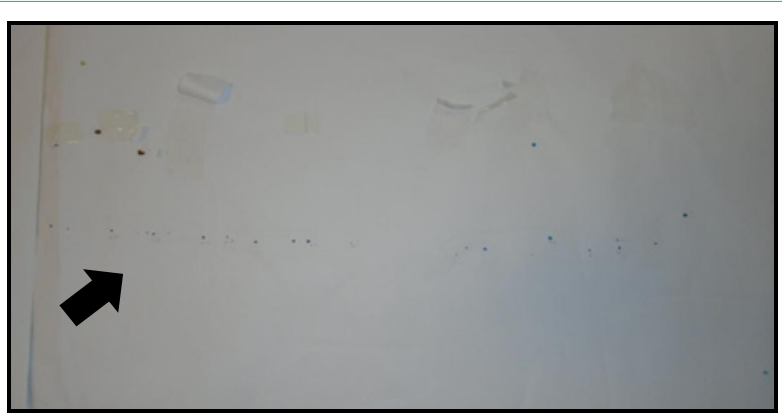

Figure 13: Linear cast-off pattern produced with blunt weapon bat.

\section{The relationship among surface area of blunt weapon and cast-off pattern}

It was observed cast-off patterns were produced only with bat which can be attributed to its high surface area and longer length, more characteristics and attributes like the porous, non-polished wooden surface texture can also be associated to the production of these patterns (Figure 13). Hammer created no cast off patterns, which can be attributed to its smaller surface area, smaller length and also to the surface of the hammer which was non porous.

\section{Estimating handedness of assailant with the help of fluid and stain distribution}

Majority of the participants used the weapons with their right hand, while only a few participants used their left hand. The resultant impact blood spatter patterns were analyzed and it was observed that right handed participants, there was more distribution of blood at the right side of the target, similarly for the left handed participants there was more distribution of blood on the left side of the target (Figure 14 and 15). This suggests that in such violent crimes involving blood and blunt weapons, the positioning and handedness of the assailant can also be estimated by comparing the difference in the amount of the bloodstains on the right and left side of the target.

\section{Discussion}

Bloodstains are one of the most important type of evidence which can be found at a crime scene, and some indications and information could be extracted from them [14]. Bloodstain pattern analysis interprets the bloodstain patterns of a crime scene in order to draw

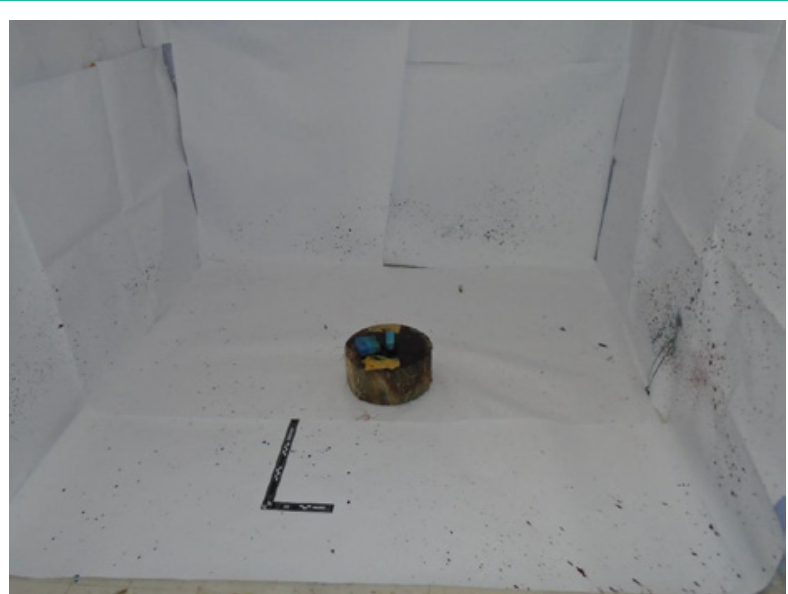

Figure 14: Right handed participants' maximum spatter formed on right wall.

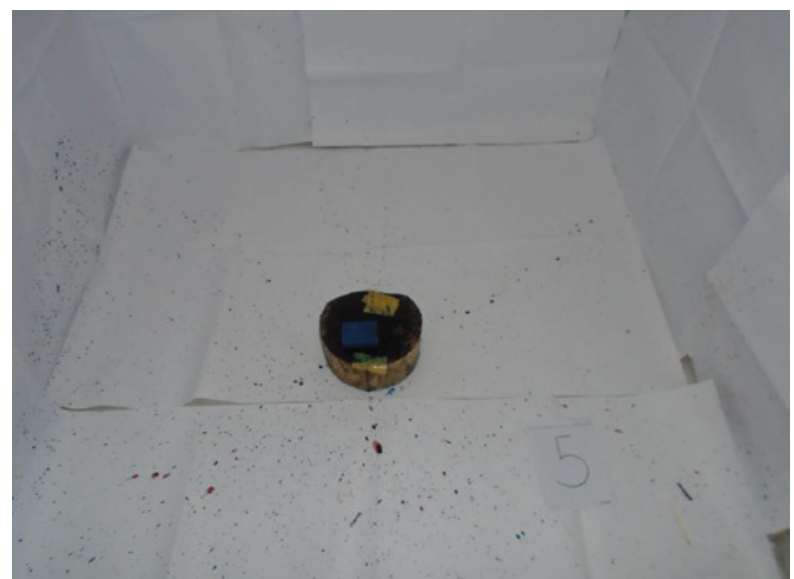

Figure 15: Left handed participants' maximum spatter formed on left wall using bat showing the relationship between handedness and blood distribution.

conclusions to support the reconstruction of the crime scene [15]. In this study impact bloodstain patterns were generated and analyzed.

In recent study an experiment was conducted in indoor setting on two test rigs to record impact beating spatters. Impact velocity and distance between blood source and target were observed in this study [16].

Petricevic and Elliot, by analyzing cast off patterns, assailant position and total amount of blood on assailant clothing, reconstructed a crime scene which involved a murder using a hammer [17].

Kunz and his colleagues analyzed cast off patterns that were produced due to the backswing motion of blood containing object [18]. William and his co-workers have explained in their study the process of cast-off pattern generation. Blood on the distal end of objects made ligaments, which eventually split into droplets to form cast-off patterns [19]. Kabaliuk and fellow scientists discussed the size of bloodstains formed with passive dripping of sharp and blunt weapons [20].

In another study, Siu and his colleagues used image analysis algorithm to analyze digital image of bloodstains produced by blunt 
force and gunshot impacts. Sponge was used as a target because of the controversy and challenges which are connected with animal experimentation as well as the reason that the sponge can hold an equal amount of blood for the experiment by every participant [21].

The focus of this research work was on the impact spatter patterns produced by using blunt weapons. The previously reported method with slightly modifications was used in the present research (Shibu and Suneetha 2015). Suneetha and Shibu analysed impact blood spatters produced by cricket bat, rock, knife and a metal rod using synthetic blood-soaked sponge as target. They analyzed two parameters bloodstain size, density of spatter [22]. In this study however, a cricket bat and a hammer were used to produce impact spatter patterns and cast off patterns using synthetic blood-soaked sponges and human blood-soaked sponges. Findings of this study can aid law enforcement agencies by providing them an indication of the approximate age group and gender of the offender and also the surface area of the blunt tool used all of which can help in narrowing down the search during investigation.

Figure 10 indicates that if the surface area of weapon and applied force are greater, then smaller stains are produced. According to this observation, it can be deduced that the impact force of females was less when compared to males therefore the stain size produced by females was larger in size as compared to the stain size produced by males. The amount of impact spatter produced by bat was also higher when compared to hammer due to the difference in surface area. Cast-off patterns were only observed to be produced by male participants using bat. It shows that production of cast-off patterns is associated with impact force and nature of the weapon used. The relationship between average stain size and the gender group as well as the age group was also observed and recorded in this study. It was observed that male participants of age group 18-30 produced more spatter with small stain size which may be due to relatively high impact force applied as compared to the other three age groups which participated in this study.

It was also observed that the cast-off patterns were only produced with bat as weapon of offence, which suggests that production of such patterns depends on the surface area of the weapon. However, it may also be dependent on the texture of the weapon. In the light of this study, it can be said that large porous wooden surface was more likely to produce cast-off patterns as compared to smaller smooth nonporous surface.

The comparative study of size of bloodstains produced from human and synthetic blood showed slight differences in the average stain size produced under similar conditions (Table 2 and Table 3) which suggests that synthetic blood is suitable to be used for future studies and for the demonstrative or training purposes. By evaluating the distribution of blood with respect to the area of all participants individually, the relationship between handedness and blood distribution was studied. it was also seen that male participants generated more amount of blood spatter with smaller bloodstain size as compared to female participants so as mentioned earlier, it can be deduced that force of impact of males is higher than females resulting in smaller bloodstains, however, more studies on this particular subject of force of impact with respect to gender can be done.

\section{Conclusion}

The classification of bloodstain patterns has been considered as a challenging aspect of bloodstain pattern analysis. In this study, a quantitative method was established using manual calculation of the average bloodstain sizes to distinguish the impact bloodstain patterns produced by different genders and age groups. The difference of behavior between the synthetic blood and human blood was also observed. Approximately 15,360 bloodstains were analyzed in this study. Results indicated that the methodology adopted to evaluate blood spatters in this study is not conclusive but is suggestive based on average bloodstain size. Male participants were found to produce more spatter with both blunt weapons (hammer and bat) and generated small bloodstain size due to higher impact force. Results showed a remarkable difference in the stain diameter produced from both kinds of fluids. Although for the demonstrative purposes in trainings as well as to study the trend of bloodstain pattern, synthetic blood can be used safely.

Studies of this nature have not been given much attention in the Pakistan as the awareness relating to this particular discipline in forensic sciences and forensic science in general can be considered relatively low as compared to other countries. This study has its significance as it gives the basic idea of how to reconstruct a crime scene through manual interpretation of bloodstains on the crime scene. This work can prove to be important in solving the criminal cases of this nature in Pakistan and the regions where the facility of automated technology to interpret and measure the bloodstains is not available or widely used. Awareness must be developed in these countries/regions about the importance of blood spatter pattern interpretation. Investigators can use this study for reconstruction of crime scenes involving impact spatter and can have an idea about gender and age group of the offender which can help in narrowing down the search of the assailant.

Improvements can be done by building on this research methodology, target sponges could be replaced other target models which are close or resemble human structure and anatomy, this would make the results even more closer to real life scenarios. It can be further probed by using blood spatter analysis software and observing further information like point of origin, position of assailant and point of convergence all of which can be deduced with the interpretation of bloodstain patterns.

\section{Acknowledgement}

The authors would like pay gratitude to Punjab Forensic Science Agency, Lahore for allowing to conduct this research work under Trace Chemistry department.

Authors gratefully acknowledge Mr. Khalid Sajjad Ferras senior Forensic Scientist at PFSA (Trace Chemistry department) whose efforts make this research work possible. Authors would like to thank Mr. Muhammad Irfan Ashiq section supervisor of Questioned Documents PFSA, for his guidance and persistent help in the research.

An appreciation also extends to personnel of Firearms and Tool marks department of Punjab Forensic Science Agency who passionately participated in the experiment. Thanks to Ms. Fazeelat, Mr. Arfan, Mr. Azeem, Ms. Faiqa, Mr. Rehan, Ms. Aisha, Ms. 
Shehnaz, Ms. Mahzaib, Mr. Irfan, Mr. Jazib and Mr. Ahsaan for their unconditional support.

\section{References}

1. Pokupic K. Blood as an Important Tool in Criminal Investigation. J Forensic Sci \& Criminal Inves. 2017; 3: 1

2. Peschel O, Kunz SN, Rothschild MA, Mützel E. Blood stain pattern analysis Forensic Sci Med Pathol. 2011; 7: 257-270.

3. Franjic S. Bloodstain Pattern Analysis Provides Evidence during Crime Scene Investigation. Int J Forensic Sci. 2019; 1: 33-38.

4. Boos K, Orr A, Illes M, Stotesbury T. Characterizing drip patterns in bloodstain pattern analysis: An investigation of the influence of droplet impact velocity and number of droplets on static pattern features. Forensic Sci Int. 2019; 30 55-66.

5. Flight $\mathrm{C}$, Jones $\mathrm{M}$, Ballantyne $\mathrm{KN}$. Determination of the maximum distance blood spatter travels from a vertical impact. Forensic Sci Int. 2018; 293: 27 36.

6. Basu N, Bandyopadhyay SK. Impact of Bloodstain Pattern Analysis in Crime Scene Investigation. Int J Adv Res. 2017; 5: 540-552.

7. James SH, Kish PE, Sutton TP. Principle of Bloodstain Pattern Analysis, Theory and Practice. Boca Raton: Taylor \& Francis Group. 2005.

8. Bandyopadhyay SK, Basu N. Interpretation of Bloodstain Pattern for Reconstruction of Crime Scene. Int Res J Comput Sci. 2015; 2: 18-22.

9. Maloney A, Campbell T, Killeen J. Visualization of Cast-off Patterns Using 3D Modelling Software. Journal of the Association for Crime Scene Reconstruction. 2011; 17: 49-56.

10. Bevel T, Gardner RM. Bloodstain Pattern Analysis: With an Introduction to Crime Scene Reconstruction. $2^{\text {nd }}$ edn. USA: CRC Press. 2002.

11. Fox JA, Fridel EE. Gender differences in patterns and trends in U.S. homicide 1976-2015. Violence Gend. 2017; 4: 37-43.
12. Stotesbury T, Illes M, Vreugdenhil A. High-speed video analysis of crown formation dynamics of controlled weapon-head impacts on to three surface types. J Can Soc Forensic Sci. 2017; 50: 64-73.

13. Oller AR. Medium Velocity Spatter Creation by Mousetraps in a Forensic Science Laboratory. Am Biol Teach. 2015; 68: 159-161.

14. Iqbal S, Ali A, Tahir MA, Tayyab M, Imran M, Feras KS. Blood droplet dynamics and its forensic implication in crime scene investigation. Foresic Res Criminol Int J. 2018; 6: 81-84.

15. Liu Y, Attinger D, De Brabanter K. Automatic Classification of Bloodstain Patterns Caused by Gunshot and Blunt Impact at Various Distances. J Forensic Sci. 2020

16. Attinger D, Liu Y, Bybee T, De Brabanter K. A data set of bloodstain patterns for teaching and research in bloodstain pattern analysis: Impact beating spatters. Data Br. 2018; 18: 648-654.

17. Petricevic S, Elliot D. Bloodstain pattern reconstruction-a hammer attack. J. Can. Soc. Forensic Sci. 2005; 38: 9-19.

18. Kunz SN, Adamec J, Grove C. Analyzing the Dynamics and Morphology of Cast-off Pattern at Different Speed Levels Using High-speed Digital Video Imaging. J. Forensic Sci. 2017; 62: 428-434.

19. Williams EMP, Graham ES, Jermy MC, Kieser DC, Taylor MC. The Dynamics of Blood Drop Release from Swinging Objects in the Creation of Cast-off Bloodstain Patterns. J. Forensic Sci. 2019; 64: 413-421.

20. Kabaliuk N, Jermy MC, Morison K, Stotesbury T, Taylor MC, Williams E. Blood drop size in passive dripping from weapons. Forensic Sci. Int. 2013; 228: 75-82.

21. Siu S, Pender J, Springer F, Tulleners F, Ristenpart W. Quantitative Differentiation of Bloodstain Patterns Resulting from Gunshot and Blunt Force Impacts. J. Forensic Sci. 2017; 62: 1166-1179.

22. Shibu G, Suneetha V. Medium Velocity Impact Blood Spatter Analysis. Int J Drug Dev Res. 2015; 7: 10-11. 